\title{
High-Order Shock-Capturing Methods for Modeling Dynamics of the Solar Atmosphere
}

\author{
Steve Bryson*
}

\author{
Alexander Kosovichev ${ }^{\dagger}$
}

May 20, 2004

\begin{abstract}
We use one-dimensional high-order central shock capturing numerical methods to study the response of various model solar atmospheres to forcing at the solar surface. The dynamics of the atmosphere is modeled with the Euler equations in a variable-sized flux tube in the presence of gravity. We study dynamics of the atmosphere suggestive of spicule formation and coronal oscillations. These studies are performed on observationally-derived model atmospheres above the quiet sun and above sunspots.

To perform these simulations, we provide a new extension of existing second- and thirdorder shock-capturing methods to irregular grids. We also solve the problem of numerically maintaining initial hydrostatic balance via the introduction of new variables in the model equations and a careful initialization mechanism.

We find several striking results: all model atmospheres respond to a single impulsive perturbation with several strong shock waves consistent with the rebound-shock model. These shock waves lift material and the transition region well into the initial corona, and the sensitivity of this lift to the initial impulse depends non-linearly on the details of the atmosphere model. We also reproduce an observed 3-minute coronal oscillation above sunspots compared to 5 -minute oscillations above the quiet sun.
\end{abstract}

Key words. coronal oscillations, spicules, rebound-shock models, high-order central-upwind schemes, balance laws, conservation laws, source terms.

AMS(MOS) subject classification. Primary 65M06; secondary 35L65.

\footnotetext{
*Program in Scientific Computing/Computational Mathematics, Stanford University and the NASA Advanced Supercomputing Division, NASA Ames Research Center, Moffett Field, CA 94035-1000; bryson@nas.nasa.gov

${ }^{\dagger}$ W. W. Hansen Experimental Physics Laboratory, Stanford University, Stanford, CA 94305-2125; sasha@quake.stanford.edu

$\ddagger$ Department of Mathematics, Stanford University, Stanford, CA 94305-2125; dlevy@math.stanford.edu
} 


\section{Contents}

1 Introduction $\quad 2$

1.1 The Solar atmosphere . . . . . . . . . . . . . . . . . 3

1.2 Previous simulations . . . . . . . . . . . . . . . . 6

2 The Model $\quad 6$

3 The Numerical Method $\quad 7$

3.1 The KNP central-upwind method . . . . . . . . . . . . . . 8

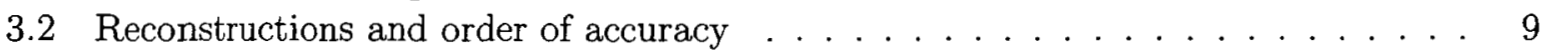

3.2 .1 A second-order reconstruction . . . . . . . . . . . . . . . . 10

3.2 .2 A third-order reconstruction . . . . . . . . . . . . . . . 11

3.3 Time integration . . . . . . . . . . . . . . . . . . 13

3.4 Hydrostatic Equilibrium . . . . . . . . . . . . . . . . . . . . 13

3.4 .1 Hydrostatic Balance . . . . . . . . . . . . . . . . . . 14

3.4 .2 Hydrostatic Initialization . . . . . . . . . . . . . . . . . . 14

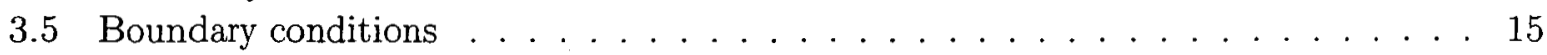

3.6 The computational domain . . . . . . . . . . . . . 16

4 Results $\quad \mathbf{1 6}$

4.1 Accuracy and comparison of the reconstructions . . . . . . . . . . . . 17

4.2 Spicule formation . . . . . . . . . . . . . . . . . . . 18

4.3 Coronal oscillations . . . . . . . . . . . . . . . . . . . 24

4.3 .1 Constant flux tube . . . . . . . . . . . . . . . . . . . . . . . . . . . . . . . . .

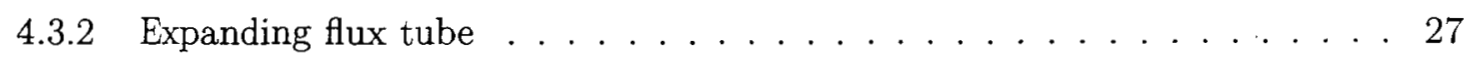

4.4 Effect of Moving the Transition Region . . . . . . . . . . . . . . . 27

5 Conclusions $\quad 29$

\section{Introduction}

This paper investigates the response of the solar atmosphere to impulsive forcing at the solar surface using initial conditions derived from observationally-based atmosphere models. We simulate the response of model atmospheres above the normal solar surface and above sunspots. Various aspects of these simulations correspond to observed phenomena on the sun. Specifically, our simulations show behavior corresponding to spicules, linear features observed in the solar 
atmosphere, and oscillations in the upper atmosphere. We find that the details of these phenomena depend on the atmosphere model in ways that correspond to the differences observed in the sun.

The dominant feature in our simulations is strong shock waves that propagate upward, lifting material from the lower atmosphere and causing oscillations of atmospheric materials. Because of these strong shocks, we perform our simulations using high-resolution central shock-capturing schemes. These schemes provide reliable approximations to solutions of the model oquations in the presence of strong shocks while avoiding spurious numerical oscillations. We extend existing methods to computational meshes which have variable grid spacing. We also use new variables defined to facilitate numerical maintenance of hydrostatic equilibrium.

One important result of our simulations is the observation that the height of the lifted material depends non-linearly on the details of the initial atmospheric model. A second result is that when we use the normal sun atmosphere model, the period of particle oscillations is in the five minute range while if we initialize our simulations using a sunspot model we see oscillations in the three-minute range. This strikingly reproduces observations of coronal oscillations in the solar atmosphere [2].

Our simulations are based on the quasi-one-dimensional Euler equations applied to an initially hydrostatic atmosphere in a magnetic flux tube. We look at flux tubes that have constant area as well as a tube whose area increases with height. This model ignores heating, radiative energy loss and thermal diffusion, as well as magnetic fields. We find it remarkable that the naive model we use produces a quantitative match with observation.

The structure of this paper is as follows: In section 1.1 we discuss basic properties of the solar atmosphere, describing its structure and summarizing the obscrved phenomena of interest to this paper. Section 2 presents the physical model that underlies our simulation. Section 3 introduces our numerical method, including a discussion of initial and boundary conditions and the computational mesh. New second- and third-order reconstructions on irregular meshes are presented in section 3.2. In section 3.4 we present a new technique for maintaining initial hydrostatic equilibrium. Section 4 presents our results, focusing on the match between our simulations and observed properties of the solar atmosphere.

\subsection{The Solar atmosphere}

The solar atmosphere is a dynamic environment with high-energy phenomena occurring on many scales. At the base of the solar atmosphere is the photosphere, the surface of sun's convective outer layer. The photosphere is roughly divided into two types of regions, quiet and active. The dominant features of the quiet photosphere are its temperature, about $5000 \mathrm{~K}$, and granulations, which are currently understood as the surface of convective cells rising from below. The surface of the photosphere has been observed to rise and fall with velocities in the range of $1 \mathrm{~km} / \mathrm{sec}$, 


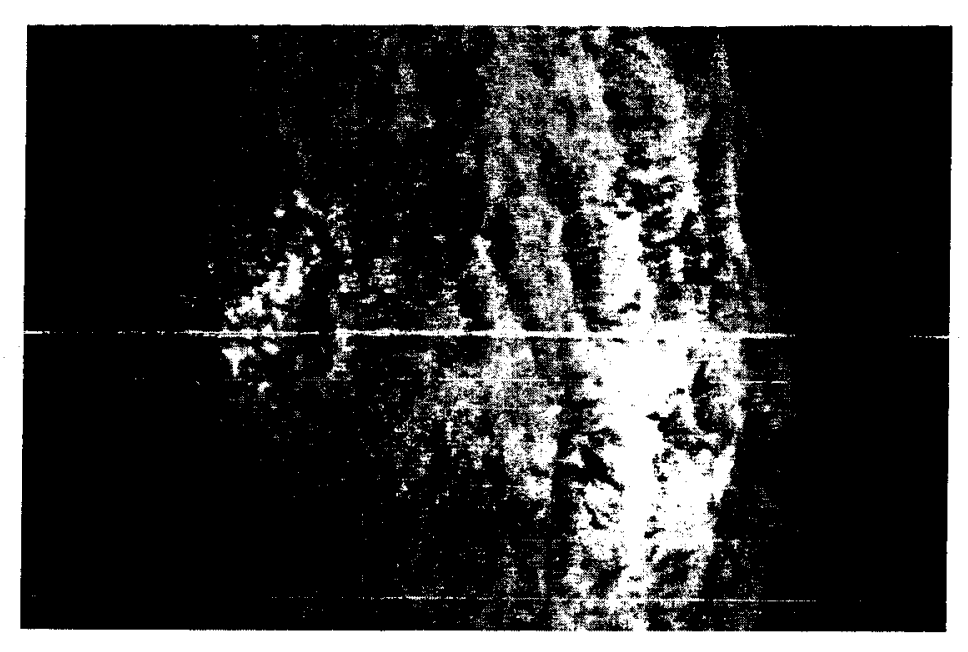

Figure 1.1: Spicules near the solar limb, seen as darker linear features against the solar surface. The spicules appear darker in this narrow-band hydrogen-alpha image because their light is blue shifted due to their motion towards the observer. Photo credit: National Solar Observatory/Sacramento Peak

and with a period of about 5 minutes. Active photosphere regions are associated with sunspots, which are regions of lower temperature, and very strong magnetic fields. Many complex and dynamic atmospheric features, such as prominences, magnetic loops and flares are associated with active regions.

Above active regions magnetic flux tubes form, where a fixed number of magnetic field lines are confined by hydrodynamic pressure into a tube. Since hydrodynamic pressure drops with increasing height, the flux tube will in general expand with altitude, requiring a quasi-1D simulation. Though many flux tubes are curved and closed, following magnetic loops, we will consider the simpler case of open magnetic flux tubes pointing straight up.

Above the photosphere, the solar atmosphere has a strongly stratified structure. The most striking feature of this stratification is the variation of temperature with height. For example above quiet regions, after slowly dropping in temperature to about $4700 \mathrm{~K}$ at about $3^{5}$ meters, at $2 \times 10^{6}$ meters there is a strong rise to about $10^{6} \mathrm{~K}$ within a few kilometers. This dramatic rise in temperature is called the transition region. The physical cause of the transition region is unknown. The solar atmosphere below the transition region is called the chromosphere, and above is the corona.

It is well known that the vertically stratified structure of the solar atmosphere implies an acoustic cutoff-frequency that corresponds to a 5-minute period. Waves with periods shorter than 5 minutes cannot effectively propagate upward in the solar atmosphere. 


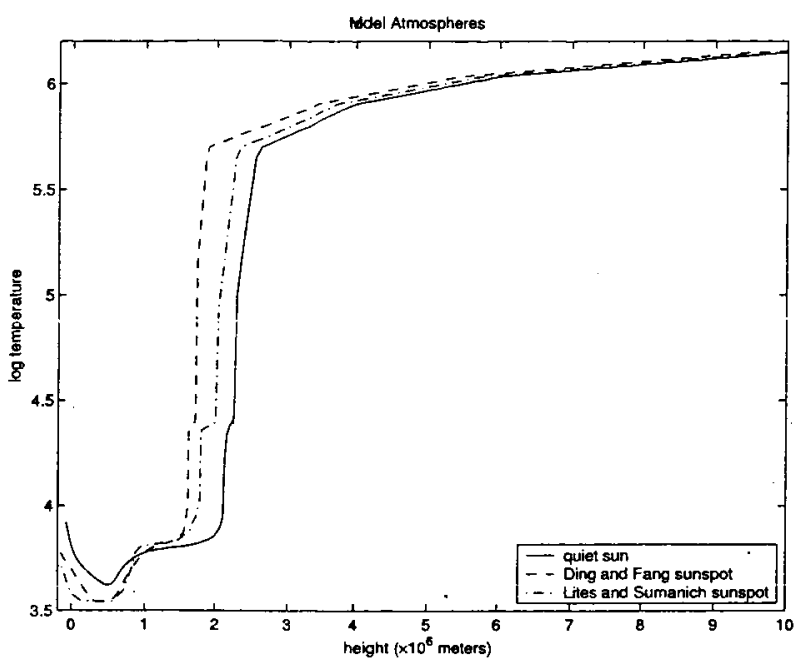

Figure 1.2: Temperature profiles in the model atmospheres we use in our simulations

One-dimensional models of the solar atmosphere have been developed, based on observed temperatures, densities and the requirement of hydrostatic equilibrium. The assumption of hydrostatic equilibrium is only approximately justified, since significant down- and up-flows have been observed. Models have been developed for the atmosphere above quiet regions [18] and sunspots [4, 11]. Fig. 1.2 shows the temperature profiles for the models that we use in our simulations.

In the simulations described in this paper we begin with an atmosphere in approximate hydrostatic equilibrium. This initial atmosphere is based on one of the model temperature profiles described above. This atmosphere is then forced by an impulse perturbation at the base of the atmosphere. There are two observed phenomena in the solar atmosphere that correspond to features that appear in our simulations.

- Spicules. Spicules $[3,14]$ are narrow near-linear features that are observed on the solar limb (see Fig. 1.1). They seem to be cooler photospheric material shooting up into the corona, and so can be modeled as an uplifting of the transition region in a magnetic flux tube. We will see such uplifting in our simulations.

- Coronal Oscillations. Particle oscillations in the corona have been observed to have periods of about 5 minutes above quiet regions and 3 minutes above sunspots [2]. We observe a similar difference in oscillation period of coronal particles when we use the quiet sun as opposed to the sunspot model atmospheres. 
We describe these observations in more detail in section 4 where we compare them directly with our simulations.

\subsection{Previous simulations}

There is a sizable literature of simulations similar to those presented in this paper, most of which attempt to model spicule formation. Eariy attempts to model spicules as a baliistic uprise of the chromosphere into the corona due to a very large $(60 \mathrm{~km} / \mathrm{sec})$ initial impulse are found in $[13,16]$. These simulations used vertical straight flux tubes. Smaller impulses leading to a rise via the rebound shock phenomenon are considered in $[1,6,7]$, some of which include expanding flux tubes. These simulations are extended in [15] by adding radiative energy loss, heating and heat conduction terms.

\section{The Model}

The quasi-one-dimensional hydrodynamic equations for material in a flux tube of area $A(x, t)$ in gravity are written in terms of density $\rho(x, t)$, velocity $u(x, t)$ and kinetic plus thermal energy $E(x, t)$ as

$$
\left(\begin{array}{c}
A \rho \\
A \rho u \\
A E
\end{array}\right)_{t}+\left(\begin{array}{c}
A \rho u \\
A \rho u^{2} \\
A(E+p) u
\end{array}\right)_{x}=\left(\begin{array}{c}
0 \\
-A p_{x}-g(x) A \rho+F(x, t) A \rho \\
-g(x) A \rho u
\end{array}\right)
$$

where $g(x)$ is the gravitational acceleration and the pressure $p(x, t)$ is given by the equation of state $p=(\gamma-1)\left(E-\frac{1}{2} \rho u^{2}\right)$ with $\gamma=5 / 3$. The temperature $T$ is given by $p=R^{\prime} \rho T$ where $R^{\prime}$ is the universal gas constant. $F(x, t)$ is a velocity forcing term which perturbs the base of the solar atmosphere in our numerical experiments. Various forcing terms will be considered.

In order to use numerical methods that were developed for conservation laws, we rewrite (2.1) as a balance law (conservation law with source)

$$
\left(\begin{array}{c}
\rho \\
\rho u \\
E
\end{array}\right)_{t}+\left(\begin{array}{c}
\rho u \\
\rho u^{2}+p \\
(E+p) u
\end{array}\right)_{x}=\left(\begin{array}{c}
-\rho u A^{-1} A_{x}-\rho A^{-1} A_{t} \\
-\rho u^{2} A^{-1} A_{x}-\rho u A^{-1} A_{t}-g(x) \rho+F(x, t) \rho \\
-(E+p) u A^{-1} A_{x}-E A^{-1} A_{t}-g(x) \rho u
\end{array}\right) .
$$

This form makes explicit that the scale of the flux tube area does not effect the dynamics: only the change in area enters the equation. Following previous authors $[6,15]$ we consider static flux tubes and have omitted the the $A_{t}$ terms from (2.2). 
For our flux tube geometry we adapt the area function of [15]. We use the functional approximation

$$
A(x)=A_{0}\left[1+b\left(a(x)-a_{0}\right)\right]^{2}
$$

where $b=3.2, a(x)=\tanh \left(s\left(x-x_{0}\right)\right)$ with $s=10^{-6}$ and $x_{0}=1.5 \times 10^{6}$, and $a_{0}=a(0)$. $\hat{A}_{0}=A(0)$ is the area of the fiux tube at the base of the ahnosphere, but dues not appear in the simulation. Therefore

$$
\frac{A^{\prime}(x)}{A(x)}=\frac{2 b s\left(1-a(x)^{2}\right)}{1+b\left(a(x)-a_{0}\right)} .
$$

\section{The Numerical Method}

In this paper we use high-order, non-oscillatory central schemes designed to solve systems of conservation laws with source terms of the form

$$
q_{t}+f(q)_{x}=S(q, x)
$$

Here $q \in \mathbb{R}^{p}$ is a $p$-dimensional solution vector, $f$ is the flux function, and $S \in \mathbb{R}^{p}$ is the $p$ dimensional vector of source terms. The solution of 3.1 may become singular in finite time, which in turn requires careful study when dealing with numerical approximations.

Our approach for solving (3.1) is based on extending the semi-discrete central-upwind scheme of Kuragnov et. al. (KNP) [9] to irregular grids. The KNP method is a simple efficient scheme with less dissipation than fully central schemes, and has desirable stability properties. Any such scheme is composed of a numerical flux, a piecewise-polynomial reconstruction and an ODE solver. The order of accuracy of the reconstruction and the ODE solver determines the order of accuracy of the method. Below we address all three ingredients, with a particular focus on our new high-order reconstructions on irregular grids.

Throughout this section we assume a one-dimensional grid with irregularly spaced nodes $x_{j}$. The distance between consecutive grids points is denoted as $\Delta x_{j}:=x_{j+1}-x_{j}$. We define $x_{j+\frac{1}{2}}:=\left(x_{j}+x_{j+1}\right) / 2$ and the cell $I_{j}=\left[x_{j-\frac{1}{2}}, x_{j+\frac{1}{2}}\right]$. For any function $f(x)$ we use the notation $f_{j}:=f\left(x_{j}\right)$. The cell average of $q$ in the cell $I_{j}$ is given by

$$
\bar{q}_{j}:=\frac{1}{x_{j+\frac{1}{2}}-x_{j-\frac{1}{2}}} \int_{x_{j-\frac{1}{2}}}^{x_{j+\frac{1}{2}}} q(x) d x .
$$


We assume that the cell-averages $\bar{q}_{j}^{n}$ are known at time $t^{n}$. The first step in the derivation of the approximate solution is to generate a piecewise-polynomial reconstruction from these cellaverages. Such a global reconstruction is defined as

$$
\tilde{q}(x)=\sum_{j} \tilde{q}_{j}(x) \chi_{I_{j}}(x),
$$

where $\chi_{I_{j}}(x)$ is the characteristic function of $I_{j}$, and $\tilde{q}_{j}(x)$ are polynomials of a suitable degree. The construction of admissible $\tilde{q}_{j}$ will be discussed in the section 3.2. For the time being, it is sufficient to assume that a reconstruction of the form (3.2) is known.

We denote the point-values of $\tilde{q}$ at the interfaces of the cell $I_{j}$ by

$$
q_{j+\frac{1}{2}}^{+}:=\tilde{q}_{j+1}\left(x_{j+\frac{1}{2}}\right), \quad q_{j+\frac{1}{2}}^{-}:=\tilde{q}_{j}\left(x_{j+\frac{1}{2}}\right) .
$$

\subsection{The KNP central-upwind method}

It is straightforward to extend the semi-discrete central scheme of [9] to irregular grids. When written in a conservative form this scheme takes the form

$$
\frac{d \bar{q}_{j}}{d t}=-\frac{H_{j+\frac{1}{2}}-H_{j-\frac{1}{2}}}{x_{j+\frac{1}{2}}-x_{j-\frac{1}{2}}}+\bar{S}_{j}
$$

where $\bar{S}_{j}$ is a discretization of the averaged value of the source term in $I_{j}$ and the numerical flux $H_{j+1 / 2}$ is given by

$$
H_{j+\frac{1}{2}}=\frac{a_{j+\frac{1}{2}}^{+} f\left(q_{j+\frac{1}{2}}^{-}\right)+a_{j+\frac{1}{2}}^{-} f\left(q_{j+\frac{1}{2}}^{+}\right)}{a_{j+\frac{1}{2}}^{+}+a_{j+\frac{1}{2}}^{-}}-\frac{a_{j+\frac{1}{2}}^{+} a_{j+\frac{1}{2}}^{-}}{a_{j+\frac{1}{2}}^{+}+a_{j+\frac{1}{2}}^{-}}\left[q_{j+\frac{1}{2}}^{+}-q_{j+\frac{1}{2}}^{-}\right] .
$$

The local speeds of propagation of information from the discontinuities on the interfaces of the cells, $a_{j \pm \frac{1}{2}}$, are given by

$$
a_{j+\frac{1}{2}}^{+}=\max \left(\lambda_{k}\left(\frac{\partial f}{\partial q}\right), 0\right), \quad a_{j+\frac{1}{2}}^{-}=\left|\min \left(\lambda_{k}\left(\frac{\partial f}{\partial q}\right), 0\right)\right|
$$

Here $\lambda_{k}\left(\frac{\partial f}{\partial q}\right)$ denote the eigenvalues of the Jacobian of $f$ evaluated at $x_{j+\frac{1}{2}}$. In the case of problem (2.2), we have

$$
a_{j+\frac{1}{2}}^{+}=\max (u-c, u, u+c, 0), \quad a_{j+\frac{1}{2}}^{-}=|\min (u-c, u, u+c, 0)|,
$$


where $c=\sqrt{\gamma p / \rho}$ is the speed of sound and all fields are evaluated at $x_{j+\frac{1}{2}}$.

We approximate the cell-average of the source term $\bar{S}_{j}$ as $S\left(\bar{\rho}_{j}, \bar{u}_{j}, \bar{E}_{j}, x_{j}\right)$. This amounts to a first-order quadrature approximation to the cell average. The $O\left(\Delta x_{j}\right)$ error from this approximation appears in the terms containing the flux tube area variation and the gravitational acceleration $g(x)$. This error is acceptable since the flux tube model (2.3) is itself only qualitative. While we compute the gravitational acceleration $g(x)$ for each cell, it is slowly varying and so is approximateiy constant in each ceii.

\subsection{Reconstructions and order of accuracy}

In this section we introduce new second- and third-order reconstructions for irregular grids that are used in the scheme (3.3). In spite of the uncertainties that exist in the model atmospheres we use for our initial conditions as well as the overall simplified nature of our model, high-order methods are valuable since they enable us to conduct long-time simulations with well resolved shock structures.

Our second-order reconstruction, when used in (3.3), is highly efficient and leads to a method that is total variation diminishing (TVD). The TVD property assures that spurious numerical oscillations will not increase from timestep to timestep. The third-order method, when used in (3.3) provides higher resolution and leads to a "number of extrema diminishing" (NED) method on regular grids. While the NED property does not guarantee that existing oscillations will not grow, it does assure us that new numerical oscillations will not be introduced. Though we have not demonstrated that the NED property holds for irregular grids, the computational mesh we use in our simulations is regular in the region of greatest interest.

The reconstruction $\tilde{q}_{j}(x)$ in the cell $I_{j}$ should satisfy the following requirements:

- Conservation.

$$
\frac{1}{x_{j+\frac{1}{2}}-x_{j-\frac{1}{2}}} \int_{x_{j-\frac{1}{2}}}^{x_{j+\frac{1}{2}}} \tilde{q}_{j}(x) d x=\bar{q}_{j} .
$$

While any linear reconstruction will have this property on a regular grid, this will not be automatically true when the grid has variable spacing.

- Accuracy. $\tilde{q}_{j}$ is an $r$ th-order accurate reconstruction, i.e. if we scale $\Delta x_{j} \rightarrow \delta \Delta x_{j}$ then the error scales as $\delta^{r}$. On a regular grid with grid spacing $\Delta x$ this amounts to

$$
\tilde{q}_{j}(x)=q(x)+O\left(\Delta x^{r}\right), x \in I_{j} .
$$

- Oscillation minimizing. Spurious numerical oscillations should be avoided. 


\subsubsection{A second-order reconstruction}

Second-order reconstructions are provided by piecewise-linear interpolants, with a different interpolant for each cell $I_{j}$. A linear interpolant that is conservative in $I_{j}$ for any $D_{j}$ is given by

$$
\tilde{q}_{j}(x)=\bar{q}_{j}+D_{j}\left(x-\left(x_{j}+\frac{1}{4}\left(\Delta x_{j}-\Delta x_{j-1}\right)\right)\right) .
$$

The discrete derivative, $D_{j}$, is taken as

$$
D_{j}=\operatorname{MinMod}\left(4 \frac{\bar{q}_{j}-\bar{q}_{j-1}}{\Delta x_{j}+2 \Delta x_{j-1}+\Delta x_{j-2}}, 4 \frac{\bar{q}_{j+1}-\bar{q}_{j}}{\Delta x_{j+1}+2 \Delta x_{j}+\Delta x_{j-1}}\right) .
$$

where the MinMod limiter is defined as

$$
\operatorname{MinMod}\left(q_{1}, \ldots, q_{n}\right)= \begin{cases}\min \left(q_{1}, \ldots, q_{n}\right), & q_{i}>0, \forall i \\ \max \left(q_{1}, \ldots, q_{n}\right), & q_{i}<0, \forall i \\ 0, & \text { otherwise }\end{cases}
$$

See [17] for a discussion of nonlinear limiters on uniform grids. The expression (3.4) now provides the required values at the cell interfaces:

$$
\begin{aligned}
& q_{j+\frac{1}{2}}^{-}=\tilde{q}_{j}\left(x_{j+\frac{1}{2}}\right)=\bar{q}_{j}+\frac{1}{4} D_{j}\left(\Delta x_{j}+\Delta x_{j-1}\right), \\
& q_{j+\frac{1}{2}}^{+}=\tilde{q}_{j+1}\left(x_{j+\frac{1}{2}}\right)=\bar{q}_{j+1}-\frac{1}{4} D_{j+1}\left(\Delta x_{j}+\Delta x_{j+1}\right) .
\end{aligned}
$$

Lemma 3.1 The reconstruction (3.4) is total variation preserving.

Proof: Our reconstruction is $R(v)=\sum_{j} \tilde{q}_{j}(x) \chi_{j}(x)$, where $\tilde{q}_{j}$ is given by (3.4). Then

$$
\begin{aligned}
T V(R(v))= & \sum_{j}\left[\left|D_{j}\right|\left(\frac{\Delta x_{j}+\Delta x_{j-1}}{2}\right)\right. \\
& \left.+\left|\bar{q}_{j}-\bar{q}_{j+1}+\frac{D_{j}}{4}\left(\Delta x_{j+1}+\Delta x_{j}\right)+\frac{D_{j+1}}{4}\left(\Delta x_{j}+\Delta x_{j+1}\right)\right|\right] .
\end{aligned}
$$

It is easy to verify that $D_{j}$ defined in (3.5) satisfies

1. $\operatorname{sgn}\left(D_{j}\right)=\operatorname{sgn}\left(D_{j+1}\right)=\operatorname{sgn}\left(\bar{q}_{j+1}-\bar{q}_{j}\right)$

2. $\frac{1}{4}\left|\left(\Delta x_{j-1}+\Delta x_{j}\right) D_{j}+\left(\Delta x_{j}+\Delta x_{j+1}\right) D_{j+1}\right| \leq\left|\bar{q}_{j+1}-\bar{q}_{j}\right|$. 
Then (assuming periodic boundary conditions)

$$
\begin{aligned}
T V(R(v))= & \sum_{j}\left[\left|D_{j}\right|\left(\frac{\Delta x_{j}+\Delta x_{j-1}}{2}\right)\right. \\
& \left.+\left|\bar{q}_{j}-\bar{q}_{j+1}\right|-\frac{\left|D_{j}\right|}{4}\left(\Delta x_{j+1}+\Delta x_{j}\right)-\frac{\left|D_{j+1}\right|}{4}\left(\Delta x_{j}+\Delta x_{j+1}\right)\right] \\
= & \sum_{j}\left|\bar{q}_{j}-\bar{q}_{j+1}\right|+\sum_{j}\left|D_{j}\right| \frac{\Delta x_{j-1}+\dot{\Delta} x_{j}}{4}-\sum_{j}\left|D_{j+1}\right| \frac{\Delta x_{j}+\Delta x_{j+1}}{4} \\
= & T V(v) .
\end{aligned}
$$

\subsubsection{A third-order reconstruction}

The third-order method from [8], based on [12], uses a linear combination of a linear interpolant $L_{j}(x)$ and a quadratic interpolant $Q_{j}(x)$ that is third-order accurate in smooth regions. Whenever a discontinuity is present, the order of the reconstruction is reduced by choosing one linear interpolant from the appropriate side of the discontinuity. This latter choice minimizes spurious numerical oscillations. The reconstruction of [8] is given in terms of the convex combination

$$
\tilde{q}_{j}(x):=\left(1-\theta_{j}\right) L_{j}(x)+\theta_{j} Q_{j}(x),
$$

where

$$
\theta_{j}:= \begin{cases}\min \left\{\frac{M_{j+\frac{1}{2}}-L_{j}\left(x_{j+\frac{1}{2}}\right)}{M_{j}-L_{j}\left(x_{j+\frac{1}{2}}\right)}, \frac{m_{j-\frac{1}{2}}-L_{j}\left(x_{j-\frac{1}{2}}\right)}{m_{j}-L_{j}\left(x_{j-\frac{1}{2}}\right)}, 1\right\}, & \bar{q}_{j-1}<\bar{q}_{j}<\bar{q}_{j+1}, \\ \min \left\{\frac{M_{j-\frac{1}{2}}-L_{j}\left(x_{j-\frac{1}{2}}\right)}{M_{j}-L_{j}\left(x_{j-\frac{1}{2}}\right)}, \frac{m_{j+\frac{1}{2}}-L_{j}\left(x_{j+\frac{1}{2}}\right)}{m_{j}-L_{j}\left(x_{j+\frac{1}{2}}\right)}, 1\right\}, & \bar{q}_{j-1}>\bar{q}_{j}>\bar{q}_{j+1}, \\ 1, & \text { otherwise }\end{cases}
$$

and

$$
\begin{aligned}
& M_{j}=\max \left\{Q_{j}\left(x_{j+\frac{1}{2}}\right), Q_{j}\left(x_{j-\frac{1}{2}}\right)\right\}, \quad m_{j}=\min \left\{Q_{j}\left(x_{j+\frac{1}{2}}\right), Q_{j}\left(x_{j-\frac{1}{2}}\right)\right\}, \\
& M_{j \pm \frac{1}{2}}=\max \left\{\frac{1}{2}\left(L_{j}\left(x_{j \pm \frac{1}{2}}\right)+L_{j \pm 1}\left(x_{j \pm \frac{1}{2}}\right)\right), Q_{j \pm 1}\left(x_{j \pm \frac{1}{2}}\right)\right\}, \\
& m_{j \pm \frac{1}{2}}=\min \left\{\frac{1}{2}\left(L_{j}\left(x_{j \pm \frac{1}{2}}\right)+L_{j \pm 1}\left(x_{j \pm \frac{1}{2}}\right)\right), Q_{j \pm 1}\left(x_{j \pm \frac{1}{2}}\right)\right\} .
\end{aligned}
$$


We now extend the method (3.6) to non-uniform grids. The linear function, $L_{j}(x)$, is taken as the conservative reconstruction (3.4) with (3.5). The quadratic function, $Q_{j}(x)$, is replaced by

$$
Q_{j}(x)=A_{j}+B_{j}\left(x-\left(x_{j}+x_{0}\right)\right)+C_{j}\left(x-\left(x_{j}+x_{0}\right)\right)^{2},
$$

where

$$
\begin{aligned}
x_{0}= & \frac{1}{4}\left(\Delta x_{j}-\Delta x_{j-1}\right), \\
A_{j}= & \bar{q}_{j}-\frac{1}{48} \gamma_{j}^{2} C_{j}, \\
B_{j}= & 2 \alpha_{j}\left[-\beta_{j}\left(\Delta x_{j-1}+3 \Delta x_{j}+2 \Delta x_{j+1}\right) \bar{q}_{j-1}+\beta_{j} \beta_{j+1} \mu_{j} \bar{q}_{j}\right. \\
& \left.+\beta_{j+1}\left(2 \Delta x_{j-2}+3 \Delta x_{j-1}+\Delta x_{j}\right) \bar{q}_{j+1}\right], \\
C_{j}= & 12 \alpha_{j}\left[\beta_{j} \bar{q}_{j-1}+\beta_{j} \beta_{j+1} \eta_{j} \bar{q}_{j}+\beta_{j+1} \bar{q}_{j+1}\right] .
\end{aligned}
$$

Here $\gamma_{j}=\Delta x_{j}+\Delta x_{j-1}$

$$
\begin{aligned}
& \alpha_{j}=\frac{1}{\left(\Delta x_{j-2}+2 \Delta x_{j-1}+2 \Delta x_{j}+\Delta x_{j+1}\right)}, \quad \beta_{j}=\frac{1}{\Delta x_{j-2}+2 \Delta x_{j-1}+\Delta x_{j}}, \\
& \eta_{j}=-\left(\Delta x_{j-2}+3 \Delta x_{j-1}+3 \Delta x_{j}+\Delta x_{j+1}\right)
\end{aligned}
$$

and

$$
\mu_{j}=\left(2 \Delta x_{j-2}+5 \Delta x_{j-1}+5 \Delta x_{j}+2 \Delta_{j+1}\right)\left(-\Delta x_{j-2}-\Delta x_{j-1}+\Delta x_{j}+\Delta x_{j+1}\right) .
$$

It is easy to verity that the interpolant (3.7) is conservative and third-order accurate on $I_{j}$. Using (3.7) we get that the point values at the cell interfaces are

$$
Q_{j}\left(x_{j+\frac{1}{2}}\right)=-\alpha_{j} \beta_{j} \gamma_{j} \gamma_{j+1} \bar{q}_{j-1}+\left(1+\frac{1}{2} \alpha_{j} \beta_{j} \beta_{j-1} \gamma_{j}\left(\gamma_{j} \eta_{j} \dot{+} \mu_{j}\right)\right) \bar{q}+\alpha_{j} \frac{\beta_{j+1}}{\beta_{j}} \gamma_{j} \bar{q}_{j+1}
$$

and

$$
Q_{j}\left(x_{j-\frac{1}{2}}\right)=\alpha_{j} \frac{\beta_{j}}{\beta_{j+1}} \gamma_{j} \bar{q}_{j-1}+\left(1+\frac{1}{2} \alpha_{j} \beta_{j} \beta_{j-1} \gamma_{j}\left(\gamma_{j} \eta_{j}-\mu_{j}\right)\right) \bar{q}_{j}-\alpha_{j} \beta_{j+1} \gamma_{j} \gamma_{j-1} \bar{q}_{j+1}
$$




\subsection{Time integration}

The time stepping is performed by either a second- or third-order TVD Runge-Kutta method from [5]. Given data $q_{j}^{n}$ at time $t^{n}$, we advance to the next time step by solving an ODE of the form

$$
\frac{d q_{j}}{d t}=F\left(q_{j}\right)
$$

A second-order TVD-RK method for (3.8) is

$$
\begin{aligned}
q^{(1)} & =q^{n}+\Delta t F\left(q^{n}\right), \\
q^{n+1} & =\frac{1}{2}\left(q^{n}+q^{(1)}+\Delta t F\left(q^{(1)}\right)\right) .
\end{aligned}
$$

A third-order TVD-RK method for (3.8) is

$$
\begin{aligned}
q^{(1)} & =q^{n}+\Delta t F\left(q^{n}\right) \\
q^{(2)} & =\frac{3}{4} q^{n}+\frac{1}{4}\left(q^{(1)}+\Delta t F\left(q^{(1)}\right)\right) \\
q^{n+1} & =\frac{1}{3} q^{n}+\frac{2}{3}\left(q^{(2)}+\Delta t F\left(q^{(2)}\right)\right)
\end{aligned}
$$

Each time step is set to

$$
\Delta t=\beta \max _{j} \frac{\Delta x_{j}}{\sigma\left(J_{j}\right)},
$$

where $\sigma\left(J_{j}\right)$ is the spectral radius of the Jacobian $J=\partial f / \partial q$ evaluated at $x_{j}$. The parameter $\beta$ is taken in our simulations as $\beta=0.9$.

\subsection{Hydrostatic Equilibrium}

We assume hydrostatic equilibrium at initialization and at the computational domain boundaries. This implies that $u=0$ and there is no time evolution so long as hydrostatic equilibrium is maintained. Therefore hydrostatic equilibrium relates pressure and density via (2.2) as

$$
\frac{d p(x)}{d x}=-g(x) \rho(x)=-\frac{g(x) p(x)}{R^{\prime} T(x)} .
$$

We use the temperature profile $T(x)$ and pressure at the bottom of the atmosphere $p(0)=$ $R^{\prime} \rho(0) T(0)$ that are given by an atmosphere model to solve (3.9) for the hydrostatic pressure profile $p(x)$. 


\subsubsection{Hydrostatic Balance}

In order to obtain numerical hydrostatic balance, we modify the model (2.2) through a change of variables. We assume that we are given an initial density $\rho_{0}(x)$ and energy $E_{0}(x)$ in hydrostatic equilibrium as described in section 3.4.2. We define the variables $\hat{\rho}:=\rho-\rho_{0}$ and $\hat{E}:=E-E_{0}$ and write the system $(2.2)$ in the mathematically equivalent form

$$
\left(\begin{array}{c}
\hat{\rho} \\
\rho u \\
\hat{E}
\end{array}\right)_{t}+\left(\begin{array}{c}
\left(\hat{\hat{\rho}}+\hat{\rho}_{0}\right) \hat{u} \\
\left(\hat{\rho}+\rho_{0}\right) u^{2}+p \\
\left(\hat{E}+E_{0}+p\right) u
\end{array}\right)_{x}=\left(\begin{array}{c}
-\left(\hat{\hat{\rho}}+\hat{\rho}_{0}\right) \hat{u} A^{-1} A_{x} \\
-\left(\hat{\rho}+\rho_{0}\right)\left(u^{2} A^{-1} A_{x}+g(x)-F(x, t)\right) \\
-\left(\hat{E}+E_{0}+p\right) u A^{-1} A_{x}-g(x)\left(\hat{\rho}+\rho_{0}\right) u
\end{array}\right),
$$

with $p:=(\gamma-1)\left(\hat{E}+E_{0}-\frac{1}{2}\left(\hat{\rho}+\rho_{0}\right) u^{2}\right)$. Here we define $u:=\rho u /\left(\hat{\rho}+\rho_{0}\right)$ and use the fact that hydrostatic equilibrium implies that the flux tube is static so $A_{t}=0$. With these new variables, $\hat{\rho}=\hat{E}=0$ as long as the system remains in hydrostatic equilibrium, i.e. satisfies (3.9) with $u=0$. We estimate $\rho_{0}\left(x_{j \pm \frac{1}{2}}\right)$ and $E_{0}\left(x_{j \pm \frac{1}{2}}\right)$ at the cell interfaces using the interpolants described in section 3.2. As described in section 3.4.2, if $\rho_{0}(x)$ is defined by (3.12), then our numerical method (2.2) will remain in hydrostatic balance as long as $u=0$.

\subsubsection{Hydrostatic Initialization}

We use (3.9) to initialize our simulations in hydrostatic equilibrium given a temperature profile specified by a Solar model. Equation (3.9) is integrated on a high-resolution grid using the standard fourth-order Runge-Kutta method, then linearly interpolated onto the actual computational grid of the simulation. This process gives an initial pressure profile $p_{0}(x)$ which is transformed into an energy profile via $E_{0}(x)=p_{0}(x) /(\gamma-1)$.

The initial density profile $\rho_{0}(x)$ is determined by the requirement of numerical hydrostatic equilibrium $u=0$ in our method (3.3). In this case we have $a_{j+\frac{1}{2}}^{+}=a_{j+\frac{1}{2}}^{-}=c_{j+\frac{1}{2}}$. Inserting these conditions into (3.3) applied to our model (2.2) (supressing the subscript 0 on $p_{0}$ for clarity), we obtain

$$
\frac{1}{2\left(x_{j+\frac{1}{2}}-x_{j-\frac{1}{2}}\right)}\left\{\left(\begin{array}{c}
\rho^{-}-\rho^{+} \\
p^{-}+p^{+} \\
E^{-}-E^{+}
\end{array}\right)_{j+\frac{1}{2}}-\left(\begin{array}{c}
\rho^{-}-\rho^{+} \\
p^{-}+p^{+} \\
E^{-}-E^{+}
\end{array}\right)_{j-\frac{1}{2}}\right\}=\left(\begin{array}{c}
0 \\
-g \rho \\
0
\end{array}\right)_{j}
$$

We therefore have hydrostatic balance in the second component if we initialize the density term in the source as

$$
\left(\rho_{0}\right)_{j}=-\frac{1}{2 g_{j}\left(x_{j+\frac{1}{2}}-x_{j-\frac{1}{2}}\right)}\left[p_{j+\frac{1}{2}}^{-}+p_{j+\frac{1}{2}}^{+}-\left(p_{j-\frac{1}{2}}^{-}+p_{j-\frac{1}{2}}^{+}\right)\right] .
$$


The first and third components of (3.11) will vanish if we use the variables defined in section 3.4.1 so long as hydrostatic equilibrium is satisfied at $x_{j}$. Therefore if we initialize $\rho$ according to (3.12), $\hat{\rho}=\rho u=\hat{E}=0$ and $E_{0}=p_{0} /(\gamma-1)$, then the method (3.3) applied to (3.10) will maintain hydrostatic equilibrium.

Remark. Eq. 3.12 is a natural discretization of the cell average using the cell interface values $p_{j \pm \frac{1}{2}}:$

$$
\frac{1}{x_{j+\frac{1}{2}}-x_{j-\frac{1}{2}}} \int_{j-\frac{1}{2}}^{j+\frac{1}{2}} g \rho d x=-\frac{1}{x_{j+\frac{1}{2}}-x_{j-\frac{1}{2}}} \int_{j-\frac{1}{2}}^{j+\frac{1}{2}} \frac{\partial p}{\partial x} d x=-\frac{1}{x_{j+\frac{1}{2}}-x_{j-\frac{1}{2}}}\left(p_{j+\frac{1}{2}}-p_{j-\frac{1}{2}}\right),
$$

which follows from the condition for hydrostatic equilibrium. Eq. 3.12 cannot be used to approximate the cell average of the source term after initialization because the system does not remain in hydrostatic equilibrium after we apply our forcing term.

\subsection{Boundary conditions}

Even though we use stretched grids, placing our boundaries at great distances as described in section 3.6, we want to limit that distance so we can use as few grid nodes as possible in the computational domain. We therefore have two issues to consider: the failure of initial hydrostatic equilibrium at the boundaries, and the problem of reflections, particularly of strong shocks. Failure of initial hydrostatic equilibrium at the boundaries will cause a wave to propagate into the domain.

Zeroth-order boundary conditions, where $q_{j}=q_{1}$ for $j<1$ and $q_{j}=q_{N}$ for $j>N$ where $N$ is the number of grid nodes, prevent reflections off the boundary but violate hydrostatic equilibrium. We therefore adapt a dynamic boundary condition approach, where the boundary conditions are hydrostatic until a high velocity value propagates from the interior to that boundary: if $u$ has not risen above a threshold $U$ since the start of the simulation then hydrostatic boundary conditions are applied. Otherwise zeroth-order boundary conditions are applied. This approach allows small reflections, which propagate more slowly than strong shocks thereby maximizing the time until the reflections enter the lower atmosphere region. For a typical simulation $U$ is taken to be 1 $\mathrm{km} / \mathrm{sec}$.

Hydrostatic boundary conditions are implemented by defining the field values in the ghost cells via linear interpolation of the high-resolution initialization grid described in section 3.4.2. This requires that the high-resolution initialization grid's domain be larger than the computational domain plus any ghost cells. 


\subsection{The computational domain}

The computational domain is divided into three regions: the lower atmosphere, which is our domain of primary interest and represents the chromosphere and lower corona, the upper corona, and a non-physical region below the solar surface. The lower atmosphere region has constant high-density grid spacing while the upper atmosphere and sub-surface regions have exponentially stretched meshes. The stretching of each stretched region is defined so that grid spacing matches that of the (non-stretched) lower atmosphere at the shared boundary, and extends to a specified distance with a specified number of nodes. In this way we can have high resolution in the region of interest and boundaries sufficiently far away such that reflected waves do not enter the lower atmosphere region during the course of the simulation. Typical simulation values place the upper boundary of the lower atmosphere at $10^{7}$ meters and the upper corona region at $5 \times 10^{8}$ meters.

The lower atmosphere domain is defined so that the solar surface is located at a coordinate value of $7.5 \times 10^{6}$ meters, which corresponds to a solar radius of $r_{\odot}=6.9599 \times 10^{8}$ meters. Thus $x=0$ corresponds to $r_{0}:=6.8849 \times 10^{8}$ meters.

The non-physical interior region. In the non-physical interior region the physics is adjusted to delay reflections from the boundary so they do not enter the physical region during the course of the simulation. We define the gravitational acceleration in the interior region to be exponentially damped with increasing depth:

$$
g(x)=\frac{G M_{\odot} \phi(x)}{r^{2}}, \quad \phi(x)=\left\{\begin{array}{cl}
1, & x \geq 0 \\
\exp \left\{-\left(\frac{x}{0.04 x_{I}}\right)^{2}\right\}, & x<0
\end{array}\right.
$$

where $M_{\odot}$ is the solar mass, $r=r_{0}+x$ and $x_{I}$ is a scale defined so that $g(x) \simeq 0$ in most of the interior region. In a typical simulation $x_{I}$ is typically taken as $-1 \times 10^{7}$ meters.

In hydrostatic equilibrium we have $d p / d x=-g \rho=-g p /\left(R^{\prime} T\right)$ so a smaller $g$ will lead to a smaller growth in $p$. We extend the temperature $T$ into the interior as a constant: $T(x)=T(0)$ for $x<0$. The result is a slower speed of sound so the bottom of the interior region can be closer, requiring fewer grid nodes, while still avoiding reflections off the lower boundary from entering the lower atmosphere region.

\section{Results}

In this section we present various results using the model and methods in this paper. We are particularly interested in the response of the solar atmosphere to a single impulse at the solar 
surface. Our focus is on simulations that bring out phenomena that resemble those observed in the solar atmosphere.

We consider the forcing term $F(x, t)$ in $(2.2)$, defined as

$$
F(x, t)=\frac{A \rho(x, t)}{\Delta t} X(x) T(t)
$$

where $A$ is a constant velocity amplitude in $\mathrm{m} / \mathrm{sec} . X$ and $T$ are square functions with respective widths $w_{x}$ and $w_{t}$ : for $\left|t-t_{0}\right| \leq w_{t}, T(t):=1$, otherwise 0 , and for $\left|x-x_{0}\right| \leq w_{x}, X(x):=1$, otherwise 0 .

In the above, $A, w_{t}$ and $w_{x}$ are tunable parameters that together determine the total energy. deposited into the atmosphere by the forcing function. We will see below that simulation results can depend strongly on these parameters, though we find phenomena matching those observed for physically reasonable values of these parameters. Our general approach will be to tune the parameters so that some aspect of the simulated phenomena matches observations and compare other aspects of the simulation. For example, we may choose parameters that cause the transition region to rise to around $5 \times 10^{6}$ meters and study the resulting particle oscillations, atmospheric profiles and shock wave development. For simplicity we assume that the solar atmosphere is entirely composed of hydrogen.

We will see below that the specific shape of the initial atmosphere may have a strong impact on the resulting phenomena.

\subsection{Accuracy and comparison of the reconstructions}

We first check the accuracy of the second- and third- order interpolants described in section 3.2 on an irregular grid. Table 4.1 shows the relative $L^{1}$ and'relative $L^{\infty}$ errors for the linear advection problem

$$
q_{t}+q_{x}=0
$$

with periodic boundary conditions. The errors are shown at $T=1$ for the periodic initial data $q(x, t=0)=\sin ^{4}(\pi x)$ on the domain $[-1,1]$ with nodes at $x_{j}=x_{j-1}+\Delta(1+\sin (2 \pi j / N) / 1.1)$, where $N$ is the number of grid nodes. Here $\Delta=1 /(N-1)$.

We now compare the quality of the second- and third-order reconstructions for our simulations of the solar atmosphere. Fig. 4.1 shows the result at $T=570$ seconds, initialized using the quiet sun model and a single-timestep square impulse at $T=0$. Two simulations, with the secondand the third-order methods, were performed with a low resolution of 200 grid nodes in the lower atmosphere region in order to highlight the difference between the second- and thirdorder methods. The other two simulations were performed with 1000 grid nodes in the lower 


\begin{tabular}{|c||c|c|c|c|c|c|c|c||}
\hline \multicolumn{1}{|c||}{} & \multicolumn{3}{c|}{ Second-order Reconstruction } & \multicolumn{3}{c||}{ Third-order Reconstruction } \\
\hline$N$ & $L^{1}$ Error & Rate & $L^{\infty}$ Error & Rate & $L^{1}$ Error & Rate & $L^{\infty}$ Error & Rate \\
\hline \hline 50 & 0.332 & - & $1.97 \times 10^{-2}$ & - & $8.69 \times 10^{-2}$ & - & $5.33 \times 10^{-3}$ & - \\
\hline 100 & 0.100 & 1.73 & $4.72 \times 10^{-3}$ & 2.06 & $1.37 \times 10^{-2}$ & 2.66 & $4.17 \times 10^{-4}$ & 3.68 \\
\hline 200 & $2.95 \times 10^{-2}$ & 1.76 & $1.02 \times 10^{-3}$ & 2.20 & $2.20 \times 10^{-3}$ & 2.64 & $3.26 \times 10^{-5}$ & 3.68 \\
\hline 400 & $8.93 \times 10^{-3}$ & 1.72 & $2.18 \times 10^{-4}$ & 2.23 & $3.79 \times 10^{-4}$ & 2.54 & $3.65 \times 10^{-6}$ & 3.16 \\
\hline
\end{tabular}

Table 4.1: Relative $L^{i}$ and $L^{\omega}$ errors and convergence rates for the reconsiructioñ on iñ iregülâr grids used in the scheme (3.3) applied to the advection equation (4.1).
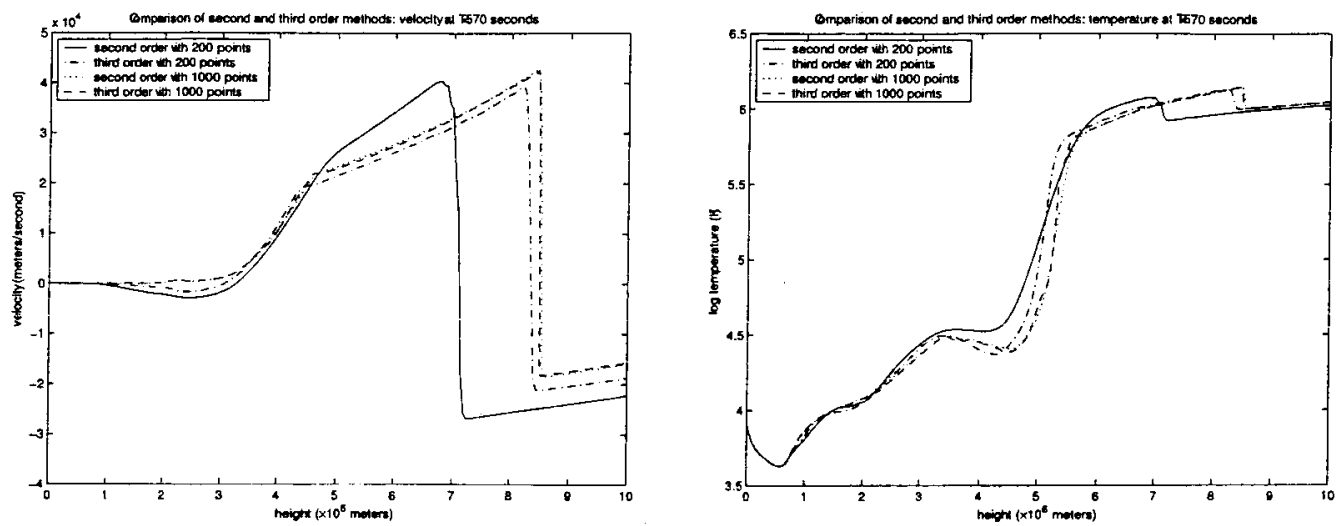

Figure 4.1: Comparison of second and third order methods. Left: Velocity. Right: Temperature.

atmosphere region. We see that there is a significant difference between the two methods in the low-resolution case, with a much smaller difference in the high resolution case. Also notable in this figure is the strength of the shock in velocity value. It is these large velocity shocks that cause the uplift and particle oscillations.

Based on these results we will perform the rest of the simulations in this section with 1000 grid nodes in the lower atmosphere region using the second order method.

\subsection{Spicule formation}

Spicules are narrow near-linear features that are observed on the solar limb [3, 14] (see Fig. 1.1). They are observed to rise with upward velocities of about $25 \mathrm{~km} / \mathrm{sec}$, have temperatures in the range of 5000 to $10,000 \mathrm{~K}$ and densities of about $3 \times 10^{-13} \mathrm{~g} / \mathrm{cm}^{3}$. Their maximum height is between $6.5 \times 10^{6}$ and $9.5 \times 10^{6} \mathrm{~m}$. Because of these relatively low temperatures and high densities, spicules can be thought of as an intrusion of chromospheric material into the corona. 

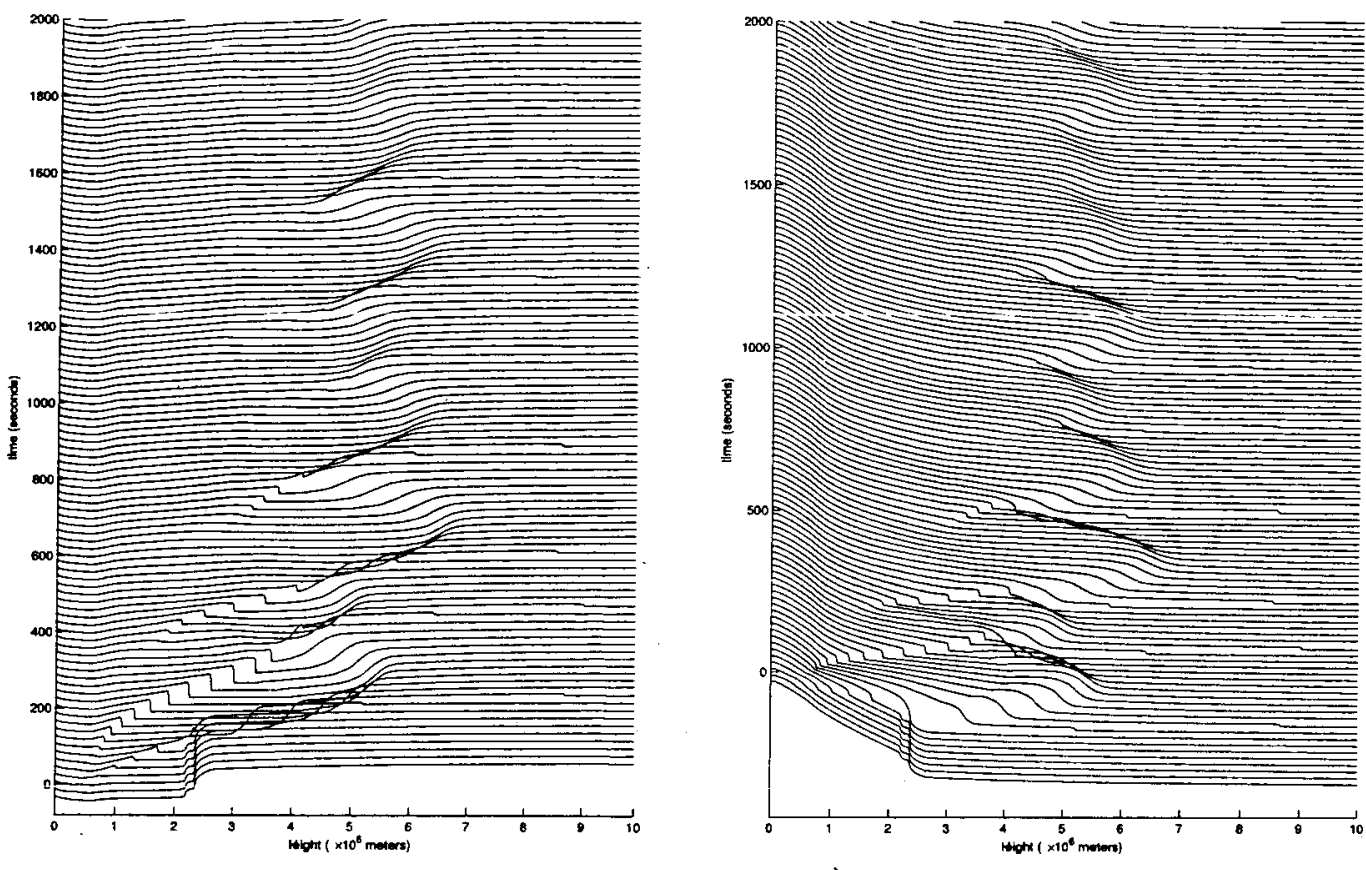

Figure 4.2: History of the temperature (left) and density (right) profile for the quiet sun model in response to a square, single-timestep $1000 \mathrm{~m} / \mathrm{sec}$ impulse.

Their linear shape can be explained as this material being channeled inside a magnetic flux tube.

We are primarily interested in the behavior of the transition region in response to impulses at the base of the atmosphere, as well as the physical conditions below the transition region at its time of maximum height. We define the position of the transition region to be where the temperature rises through $200,000 \mathrm{~K}$. We compare simulations using the three atmosphere models with both constant and expanding flux tubes. We do this despite the fact that spicules are not observed above sunspots in order to study the dependence of spicule formation on the atmosphere model.

The values of $w_{t}$ and $w_{x}$ in the impulse function $F(x, t)$ (see the introduction to section 4) are chosen so that for $A=1000 \mathrm{~m} / \mathrm{sec}$ the transition region is lifted to just above $5 \times 10^{6}$ meters in the quiet sun atmosphere. These values of $w$ are different for the case of constant flux tube width vs. an expanding flux tube. For a constant flux tube we use $w_{t}=0.065$ seconds and $w_{x}=1.4 \times 10^{5}$ meters. For expanding flux tubes significantly longer impulses were required to generate the required lift of the transition region: in this case $w_{t}=0.325$ seconds.

The dominant feature of our simulations is strong initial and rebound shock waves that re- 
peatedly appear for as long as 30 minutes after the single initial impulse. An example of the effect of these shocks on the quiet sun model is shown in Fig. 4.2. The shock trajectories for $A=1000 \mathrm{~m} / \mathrm{sec}$ are shown for the various model conditions in Fig. 4.3. We see a characteristic increase in speed as the shock crosses the transition region from the chromosphere to the corona. As time passes, this increase in speed occurs at higher altitudes as the transition region rises. The expanding flux tube also shows short-lived downward propagating shocks below the trañinitioñ regionion. As seeni in Tig. 4.1, shochs in velocity reach amplitudes as high as $10 \mathrm{~km} / \mathrm{sec}$ by the time they reach the transition region. This causes a dramatic uplift, i.e. lifting material and the transition region well into the initial corona. We track the motion of atmospheric material by seeding test particles at specific heights, then tracking their motions throughout the simulation. The position $x(t)$ of these particles is updated at each time step by solving the ODE $x^{\prime}(t)=u(x, t)$ via the standard fourth-order Runge-Kutta method, where $u(x, t)$ is the velocity component of the approximated solution. The resulting particle histories is shown in Fig. 4.4.

We see immediately from Fig. 4.4 that the Lites-Skumanich sunspot model is much more responsive to the initial perturbation, with much higher uplift than the other two models. This is striking since, as seen in Fig. 1.2, the Lite-Skumanich model is in some sense intermediate between the other two models. This demonstrates a sensitive non-linear dependence of the ability to form spicules on the details of the atmosphere model. This behavior is also evident in Fig. 4.5, which shows the maximum height of the transition region for varying impulse amplitudes. The three models exhibit a near-linear dependence on impulse amplitude for the constant flux tube, while for the expanding flux tube the Lites-Skumanich sunspot model shows a greater sensitivity. The Ding-Fang sunspot model is least responsive, requiring stronger impulses to rise to desired heights,. We see that it is not difficult to raise the transition region to $5 \times 10^{6}$ meters, the height appropriate for spicules, in all cases, though we remind the reader that we tuned our parameters for such a rise in the quiet sun model.

Fig. 4.6 shows estimates of the speed of the transition region in its initial rise for varying impulse amplitudes. This estimate is defined as the height of the first local maximum divided by the time taken to reach that height from first motion. In all cases the speed of the rise increases approximately linearly with amplitude. For the constant flux tube with impulse amplitudes of $1000 \mathrm{~m} / \mathrm{sec}$ we see rise speeds of about $22 \mathrm{~km} / \mathrm{sec}$ for the quiet sun and Lites-Skumanich sunspot models, while the Ding-Fang sunspot model shows a slower rise. For an expanding flux tube we see somewhat slower rise speeds, about $18 \mathrm{~km} / \mathrm{sec}$ for the quiet sun and Lites-Skumanich models and $1000 \mathrm{~m} / \mathrm{sec}$ impulse.

To examine the physical conditions below the transition region at time of maximum rise we consider impulse amplitudes that lift the transition region just above $5 \times 10^{6}$ meters, which is different for each atmosphere model. The temperature and density profiles for these cases are shown for the three atmosphere models and both flux tube types in Fig. ??. The properties observed from this figure are summarized in table 4.2 , where we see that our simulations match 

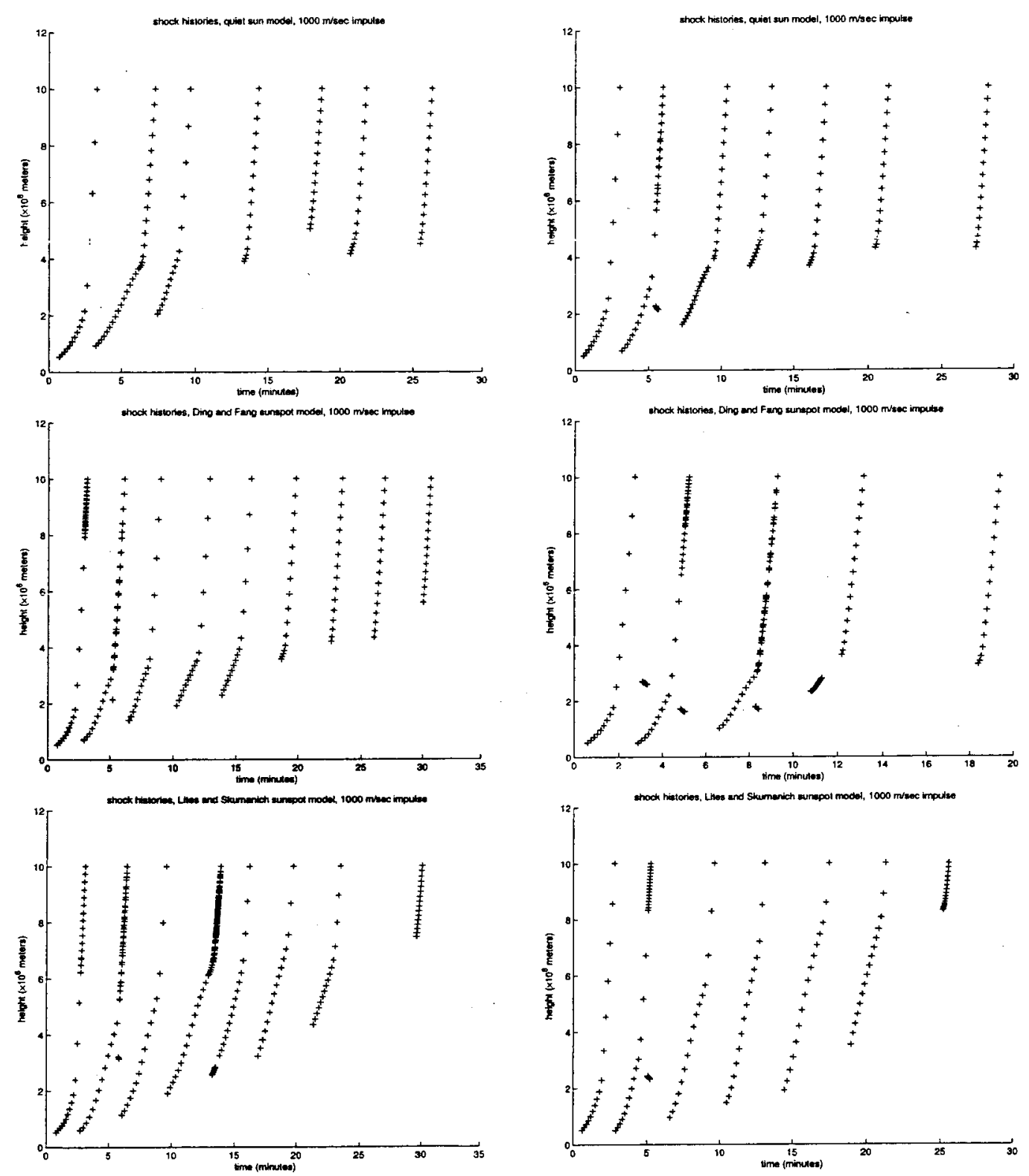

Figure 4.3: The trajectories of the velocity shocks for an impulse amplitude of $1000 \mathrm{~m} / \mathrm{sec}$. Left: Constant flux tube. Right: Expanding flux tube. Top: Quiet sun model. Middle: Ding-Fang sunspot model. Bottom: Lites-Skumanich sunspot model. Note the downward propagating shocks in the expanding flux tube. 

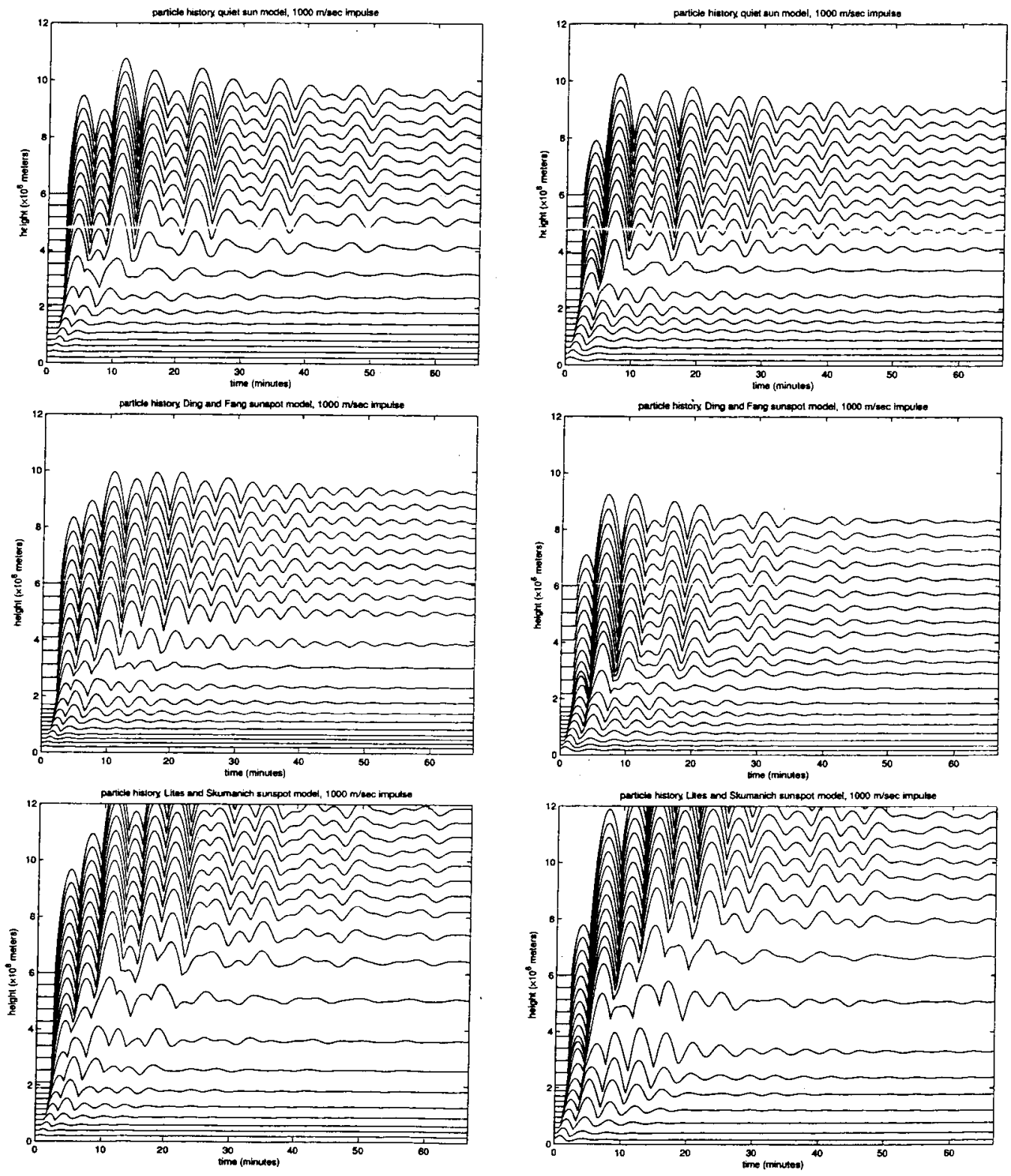

Figure 4.4: Particle histories for a square, single-timestep impulse. Left: Constant flux tube. Right: Expanding flux tube. Top: Quiet sun model. Middle: Ding-Fang sunspot model. Bottom: LitesSkumanich sunspot model. 

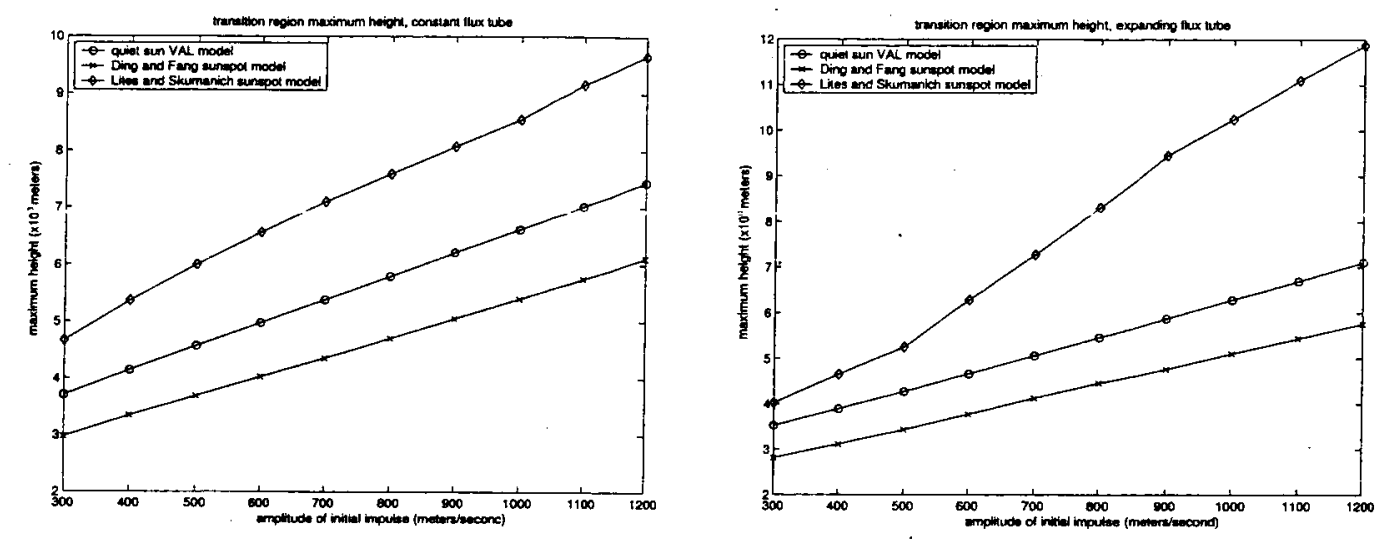

Figure 4.5: Maximum height of the transition region for various surface velocity perturbation amplitudes. Left: Constant flux tube. Right: Expanding flux tube.
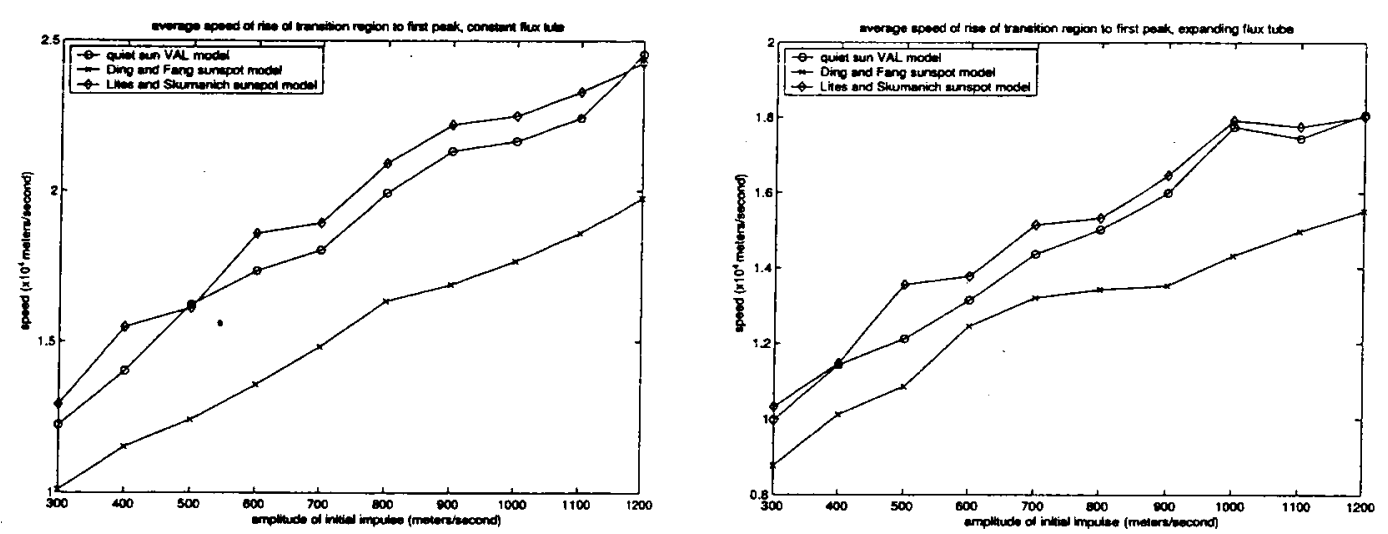

Figure 4.6: Average speed of rise of the transition region from initial position to first peak height vs. impulse amplitude. Left: Constant flux tube. Right: Expanding flux tube. 
the observations remarkably well.

\begin{tabular}{|c||c|c|c|c|c|c|c|}
\hline & & \multicolumn{3}{|c|}{ Constant flux tube } & \multicolumn{3}{c|}{ Expanding flux tube } \\
\hline & Obseved & VAL & $D F$ & $L S$ & VAL & DF & LS \\
\hline \hline Temperature $(\mathrm{K})$ & $0.5 \times 10^{4}-10^{4}$ & $2 \times 10^{4}$ & $2 \times 10^{4}$ & $2 \times 10^{4}$ & $10^{4}$ & $10^{4}$ & $3 \times 10^{4}$ \\
\hline Density $\left(\mathrm{g} / \mathrm{cm}^{3}\right)$ & $3 \times 10^{-13}$ & $2 \times 10^{-13}$ & $10^{-12}$ & $10^{-12}$ & $3 \times 10^{-13}$ & $2 \times 10^{-12}$ & $10^{-12}$ \\
\hline
\end{tabular}

Table 4.2: Temperaiure and density below. the trainsition regionin at time of mâximininin rise based on Fig. 4.7 compared with observed spicule properties.

\subsection{Coronal oscillations}

Recent observations in [2] have detected a notable difference in the period of oscillation of coronal particles above sunspots compared to periods above the quiet sun. Above sunspots, the observed oscillations have periods clustered around 3 minutes, while above the quiet sun the periods are clustered near 5 minutes (see Fig. 4.8).

In our simulations, we find a similar relationship between period of oscillation of particles in the corona (above the transition region). As seen in Fig. 4.4, coronal material exhibits an oscillatory behavior after being lifted by the shocks. Due to the multiple shocks this behavior is complex, but there are dominant modes of oscillation.

For all model atmospheres, the period of oscillations of our coronal particles depends on the amplitude of the initial impulse, though the details of this dependence differs from model to model. We study of this dependence by fixing the impulse parameters $w_{t}$ and $w_{x}$ for each model and varying $A$. We use the same values for $w_{t}$ and $w_{x}$ as in our spicule study.

The coronal oscillations found in our simulations are summarized in Fig. 4.9 and discussed in detail below. Example power spectra for $A=1000 \mathrm{~m} / \mathrm{sec}$ are shown in Fig. 4.10. We find that there is a significant difference between the case of a constant width flux tube and an expanding flux tube.

\subsubsection{Constant flux tube}

In Fig. 4.9 we see that when we use the quiet sun model atmosphere as our initial condition, we find a monotonically increasing relationship between the initial impulse amplitude and the period of coronal oscillations. The period of peak oscillation in this case ranges from 4 minutes for a $200 \mathrm{~m} / \mathrm{sec}$ impulse to 6.5 minutes for a $1200 \mathrm{~m} / \mathrm{sec}$ impulse.

When we use the Ding-Fang model atmosphere above sunspots, we find a more complex situation. For low-amplitude 200 to $400 \mathrm{~m} / \mathrm{sec}$ impulses, we find coronal oscillations with periods 

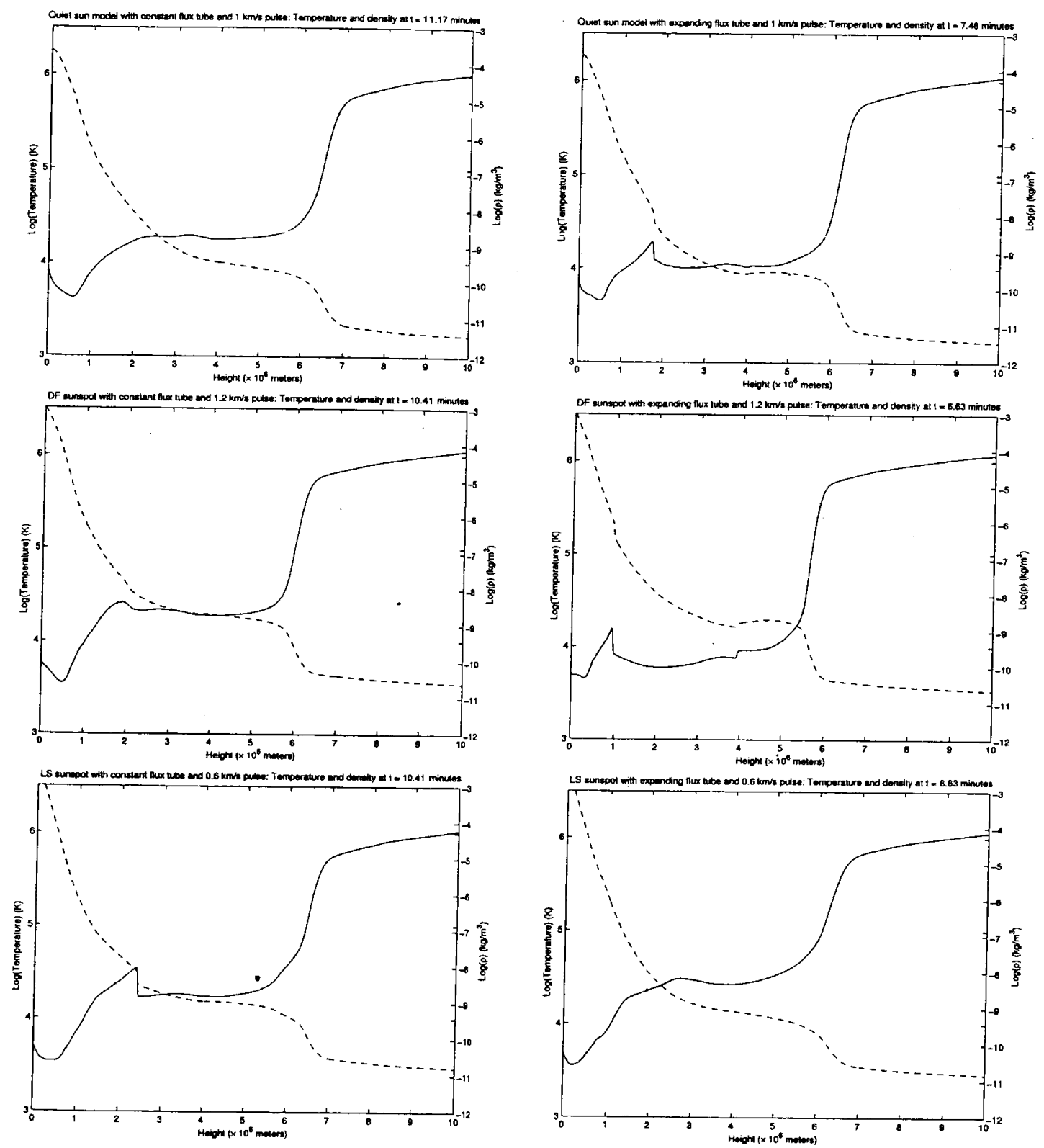

Figure 4.7: The temperature (solid line) and density (dashed line) profiles at the time of maximum transition region height in response to a square, single-timestep impulse. Left: Constant flux tube. Right: Expanding flux tube. Top: Quiet sun model. Middle: Ding-Fang sunspot model. Bottom: Lites-Skumanich sunspot model. 

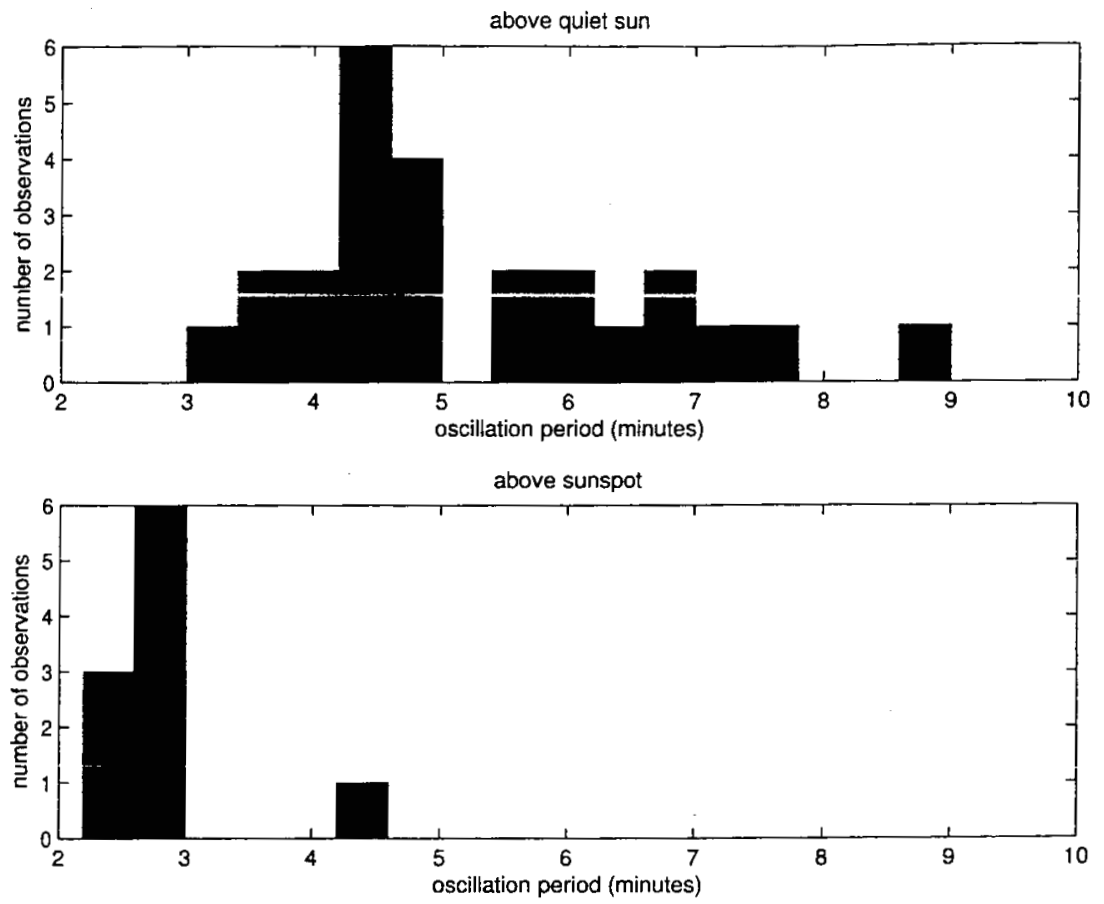

Figure 4.8: Histograms of observed periods of coronal oscillations. Top: Above the quiet sun. Bottom: Above sunspots.
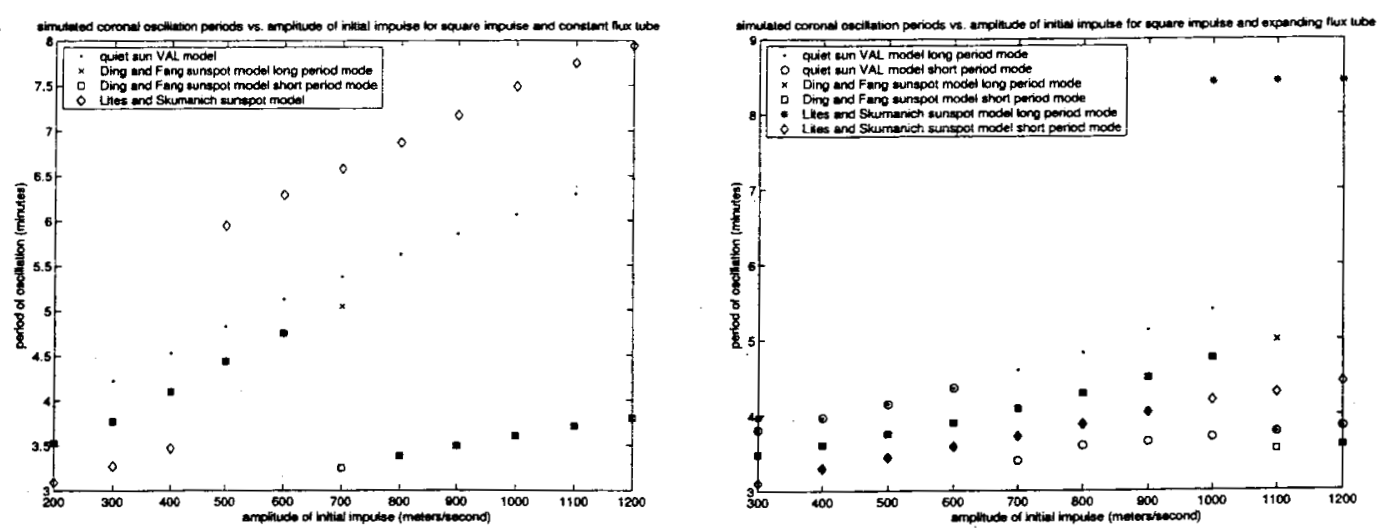

Figure 4.9: The simulated periods of oscillations for the three atmosphere models and various surface velocity perturbation amplitudes. Left: Constant flux tube. Right: Expanding flux tube. 
in the 3.5 to 5 minute range. For an amplitude of $700 \mathrm{~m} / \mathrm{sec}$, we find a bimodal regime with one mode of oscillation at about 3.2 minutes, and the other at about 5 minutes. For amplitudes from 900 to $1000 \mathrm{~m} / \mathrm{sec}$ the longer-period mode is significantly damped, and the dominant mode has periods around 3.5 minutes.

The Lites-Skumanich sunspot model shows a monotonic dependence on impulse amplitude, but the dominant mode of oscillation jumps from between 3 and 3.5 minutes, for amplitudes ranging from 200 to $500 \mathrm{~m} / \mathrm{sec}$, to 6 minintes for a $500 \mathrm{~m} / \mathrm{sec}$ impulse. The dominant period of oscillation increases almost linearly for impulse amplitudes from 500 to $1200 \mathrm{~m} / \mathrm{sec}$.

We see that for a constant width flux tube the Ding-Fang sunspot model and the quiet sun model shows coronal oscillations that are consistent with observed coronal oscillations above sunspots and above the quiet sun.

\subsubsection{Expanding flux tube}

For expanding flux tubes we see in Fig. 4.9 a bimodal behavior for all the atmosphere models. In the quiet sun model we find the dominant mode of oscillation increasing in the four minute range for impulse amplitudes ranging from 300 to $600 \mathrm{~m} / \mathrm{sec}$. For impulses between 700 and 1000 $\mathrm{m} / \mathrm{sec}$ we see bimodal behavior, with the longer mode's period rising to over 5 minutes, and a shorter mode in with a near-constant period of 3.5 minutes. For higher amplitudes the slower mode is damped and the dominant mode is just over 3.5 minutes.

In the Ding-Fang sunspot model we have a single dominant mode for impulse amplitudes between 300 and $1000 \mathrm{~m} / \mathrm{sec}$, with the period rising monotonically from 3.5 to just under 5 minutes. At an amplitude of $1100 \mathrm{~m} / \mathrm{sec}$ we see bimodal behavior, with a slow mode of 5 minutes and a fast mode of 3.5 minutes. At $1200 \mathrm{~m} / \mathrm{sec}$ the slow mode is damped and the dominant mode is about 3.5 minutes.

For the Lites-Skumanich sunspot model, we see a bimodal behavior at the lower amplitude of $300 \mathrm{~m} / \mathrm{sec}$, with 3 and 3.8 minute modes, and amplitudes of $1000 \mathrm{~m} / \mathrm{sec}$ and above. In between we have a dominant mode which increases from about 3.3 minutes to 4 minutes. For amplitudes of $1000 \mathrm{~m} / \mathrm{sec}$ and above we have a very slow mode of over 8 minutes and a fast mode of just over 4 minutes.

\subsection{Effect of Moving the Transition Region}

In an attempt to understand the role the location of the transition region plays in the above results we present simulations in which the quiet sun model is scaled so that the transition region has various heights. We use the standard square $1000 \mathrm{~m} / \mathrm{sec}$ impulse for this study.

In Fig. 4.11 we see show the dependence of the maximum transition region height on the initial transition region height. We see that there is a non-linear dependence, where initially 

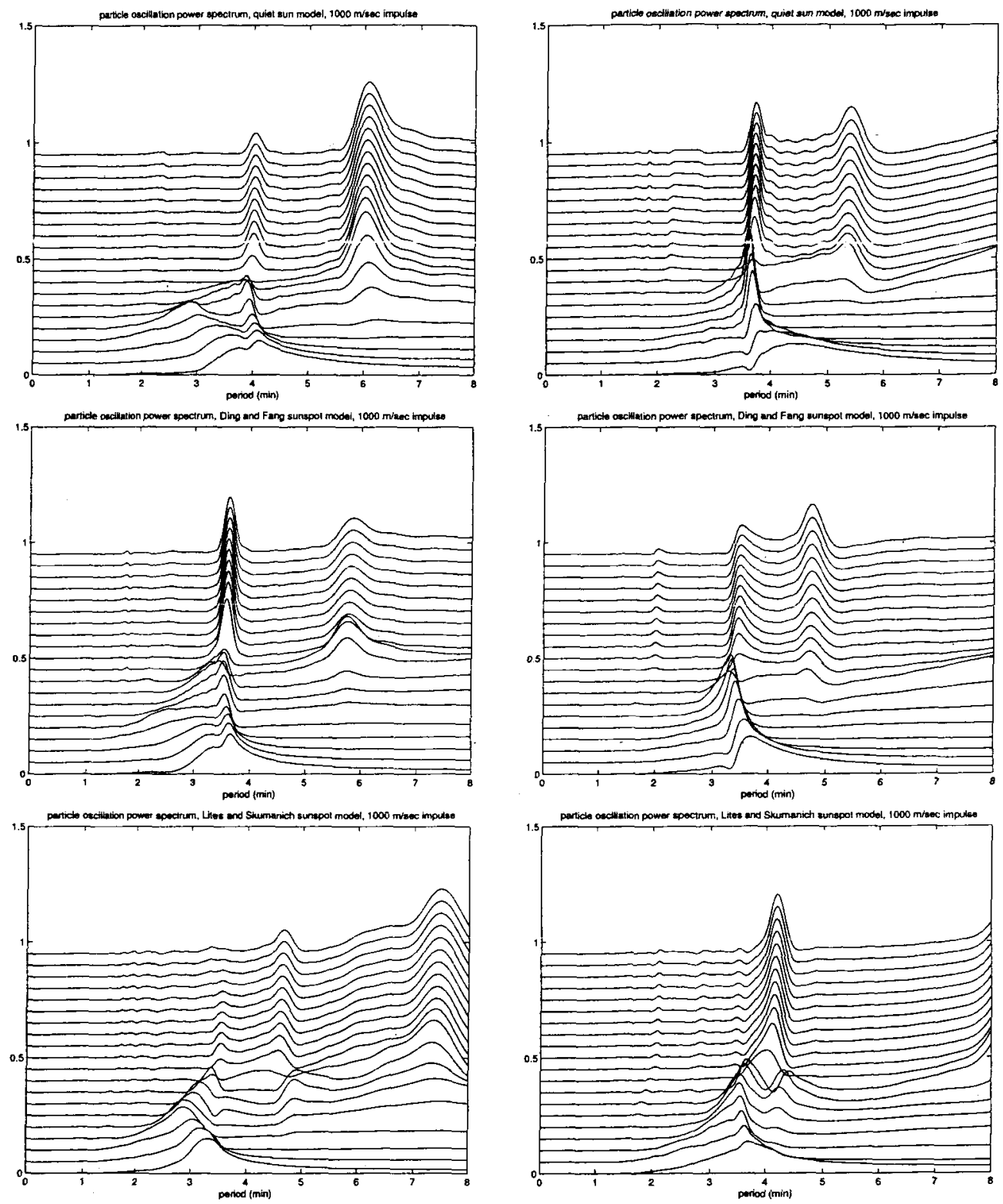

Figure 4.10: Power spectra of particle oscillations for a square, single-timestep impulse. The spectra are stacked in order of the height of the particles. Left: Constant flux tube. Right: Expanding flux tube. Top: Quiet sun model. Middle: Ding-Fang sunspot model. Bottom: Lites-Skumanich sunspot model. 


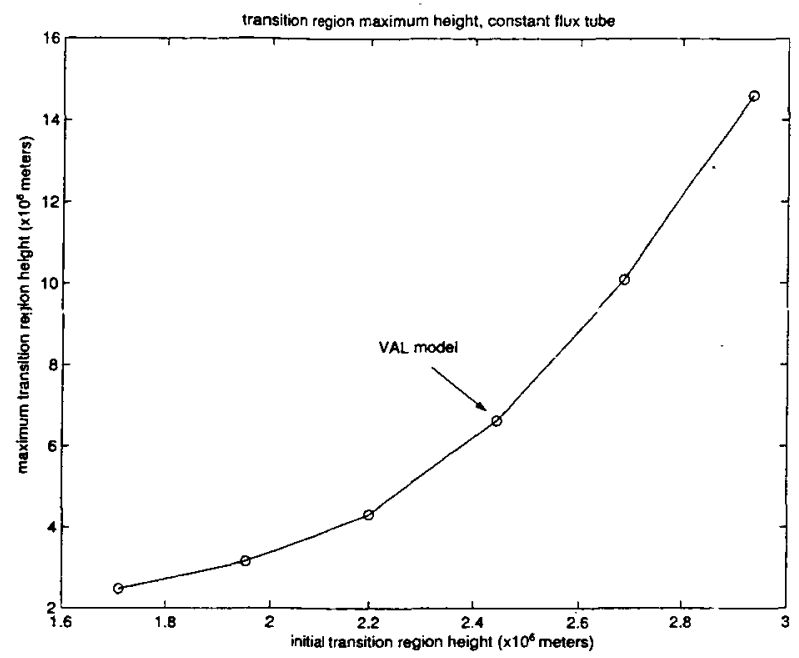

Figure 4.11: Maximum height of the transition region in response to a square impulse of $1000 \mathrm{~m} / \mathrm{sec}$ as a function of the initial transition region height in a scaling of the quiet sun model.

higher transition regions get lifted much higher. One possible explanation is the fact that the rising shocks increase in strength as the density decreases in the hydrostatic atmosphere, which results in higher velocities by the time the shock reaches the transition region.

\section{Conclusions}

In this paper we used a high-order shock-capturing scheme to study the response of various one-dimensional model solar atmospheres to a single impulse at the solar surface. Our treatment includes an extension of existing methods to irregular grids and a new technique which numerically maintains initial hydrostatic balance. In spite of the simplicity of our model we find a remarkable correspondence between our results and observation: 3-minute coronal oscillations above sunspots compared with 5-minute periods above quiet regions observed in [2] and appropriate spicule properties $[3,14]$. These results suggest that a viable mechanism of spicule formation is large velocity shocks in response to surface impulses. We further find that the lifting of material in response to an initial impulse depends non-linearly on the details of the initial atmosphere model.

The next steps in our study include adding energy loss and heating to our model following [15]. This will allow us to study the atmospheric response to continuous surface forcing. Extensions to two dimensional studies will follow, where the model will be extended to the 
magnetohydrodynamic equations.

Acknowledgment: The work of D. Levy was supported in part by the National Science Foundation under Career Grant No. DMS-0133511.

\section{References}

[1] Andreev, A., S., Kosovichev, A. G., On a Mechanism of Spicule Formation by Shock Waves in Magnetic Tubes, Astronomy Letters, 403, (1994), pp.323-326.

[2] De Moortel, I., Ireland, J., Hood, A. W., Walsh, R. W., The Detection of 3 \& 5 Minute Period Oscillations in Coronal Loops, Astronomy and Astrophysics, 387, (2002), pp.L13L16.

[3] de Pontieu, B., PhD Thesis, (1996), University of Ghent, Belgium.

[4] Ding, M. D., Fang, C., A Semi-Emperical Model of Sunspot Penumbra, Astronomy and Astrophysics, 235, (1989), pp.204-212.

[5] Gottlieb S., Shu C.-W., Tadmor E., Strong stability-preserving high order time discretization methods, SIAM Review, 43 (2001), pp.89-112.

[6] Hollweg, J. V., On the Origin of Solar Spicules, ApJ, 257, (1982), pp.345-353.

[7] Kosovichev, A. G., Popov, Yu. P., The Computation of One-Dimensional Non-Stationary Problems of Gravitational Gas Dynamics, USSR Comput. Maths. Math. Phys., 19, (1998), pp.168-175.

[8] Kurganov, K., Petrova, G., A Third-Order Semi-Discrete Genuinely Multidimensional Central Scheme for Hyperbolic Conservation Laws and Related Problems, Numerische Mathematik, 88, (2001), pp.683-729.

[9] Kurganov, K., Noelle, S., Petrova, G., Semi-Discrete Central-Upwind Schemes for Hyperbolic Conservation Laws and Hamilton-Jacobi Equations, SIAM J. Sci. Comp., 23, (2001), pp.707-740.

[10] Levy, D., Puppo, G., Russo, G., A Fourth Order Central WENO Scheme for MultiDimensional Hyperbolic Systems of Conservation Laws, SIAM J. Sci. Comp., 24, (2002), pp.480-506. 
[11] Lites, B. W., Skumanich, A., A Model of a Sunspot Chromosphere Based on OSO 8 Observations, ApJS, 49, (1989), pp.293-316.

[12] Liu, X.-D. L., Osher, S., Nonoscillatory high order accurate self-similar maximum principle satisfying shock capturing schemes I, SIAM J. Numer. Anal., 33, no. 2 (1996), pp.760-779.

[13] Shibata, K., Suematsu, Y., Why are Spicules Absend Over Plages and Long Under Coronal Holes?, Solar Physics, 78, (1982), pp.333-345.

[14] Sterling, A. C., Solar Spicules: A Review of Recent Models and Targets for Future Observations, Solar Phys., 196, (2000), pp. 79-111.

[15] Sterling, A. C., Mariska, J. T., Numerical Simulations of the Rebound Shock Model for Solar Spicules, ApJ, 349, (1990), pp. 647-655.

[16] Suematsu, Y., Shibata, K., Nishikawa, T., Kitai, R., Numerical Hydrodynamics of the Jet Phenomena in the Solar Atmosphere I. Spicules, Solar Physics, 75, (1982), pp.99-118.

[17] Sweby P.K., High Resolution Schemes Using Flux Limiters for Hyperbolic Conservation Laws, SIAM J. Numer. Anal., 21, (1984), pp. 995-1011.

[18] Vernazza, J. E., Avrett, E. H., Loeser, R., Structure of the solar chromosphere. III - Models of the EUV brightness components of the quiet-sun, ApJS 45, (1981), pp. 635-725. 